\title{
Visualising the 'real' and the 'fake': Emotion work and the representation of orgasm in pornography and everyday sexual interactions
}

\section{Abstract}

Visual representations of orgasm - whether in the flesh or mediated through a screen are produced in a context of intense uncertainty about whether what is being seen represents an authentically experienced bodily event. Despite detailed scientific scrutiny and close attention to bodily signs, the authenticity of women's orgasm remains a site of cultural anxiety and contested gender politics. This uncertainty is exacerbated by the construction of female orgasm as inherently invisible or un-see-able, and 'faking' orgasm as a prevalent social practice. Drawing on existing literature from psychology, sociology and porn studies, this theoretical paper explores the problematic of visually representing orgasm in the context of these uncertainties, and examines how the distinction between the 'real' and the 'fake' is structured by discourses of authenticity. Pornography and everyday sexual interactions provide ideal contexts for exploring the practices of producing and consuming visual representations of embodied experience since both necessitate a see-able orgasm which consumers/lovers can read as 'real'. This paper demonstrates that considerable interpretative work is necessary to read the female body as authentically orgasmic in the context of cultural uncertainty, and that distinctions between the 'real' and the 'fake' are continually reworked. Drawing on the contrast between 'surface' and 'deep' acting (Hochschild 1983) I argue that the 
distinction between the 'real' and the 'fake' cannot be established by recourse to unmediated bodily experience, and instead researchers should consider how and when this distinction has traction in the world and the implications of this for gendered power relations, subjectivities and practices.

Key words: pornography; orgasm; emotion work; embodiment 


\section{Visualising the 'real' and the 'fake': Emotion work and the representation of orgasm in pornography and everyday sexual interactions}

In the context of a cultural fascination with reading the interiority of the person from the exteriority of the body (Ward 2010), the invisibility of women's orgasm and the lack of a definitive symbol of female sexual climax equivalent to male ejaculation, produces uncertainty about the authenticity of orgasm and the necessity for visual representation ${ }^{\text {. }}$ This uncertainty is intensified by the articulation of 'faking' orgasm as a common female practice, with between $50-65 \%$ of women reporting that they have, at one time or another faked an orgasm (Schaefer 1973, Hite 1976, Darling and Davidson 1986, Weiderman 1997, Muehlenhard and Shippee 2010). Orgasm is supposed to be a moment of truth (Potts 2002), but the practice of faking orgasm ensures that the veracity of women's orgasm is continually contested. The practice of faking is typically regarded as a 'complex emotional response to the intensely patriarchal culture in which women have sex' (Fahs 2011, p. 63), which produces an obligation to give a 'noisy and exaggerated display' of orgasmic ecstasy to meet these cultural demands (Roberts et al. 1995, p. 528). With so much cultural meaning loaded onto the presence of orgasm, the necessity of producing an orgasm which is see-able, and documenting the bodily signs by which orgasm can be definitively 'read off' from women's bodies, becomes a cultural preoccupation. According to Patton (1989, p. $105 \& 107)$ female sexual pleasure is typically depicted through facial expressions 'the transcendent glazedover eyes, lips glistening and slightly parted, head thrown back', but remains 'a signification in the face of an event occurring elsewhere, if at all'. Visual representations of female 
pleasure rely on the performance or display of orgasm through facial expressions or bodily movements - yet at the same time the widespread practice of feigning orgasm renders this performance unreliable. Taken together these two issues - the invisibility of female orgasm and women's propensity to fake - pose a considerable challenge to distinguishing between the real and the fake in the visual representation, and recognition, of orgasm.

The sensory aspects of felt embodiment are typically understood as the key to distinguishing between the real and the fake - an orgasm is either authentically felt in the body, or deliberately performed through the body. Jackson and Scott (2007) have recently called for a theorisation of orgasm as both embodied and socially mediated claiming that 'even this most individual, "private", "physical" experience is always also social' (p. 96). To recognise a bodily experience as an orgasm, they argue, requires considerable interpretative work as we draw on cultural scripts to interpret the bodily sensations provided by our senses. Orgasm does not just happen; it must be learned by developing an understanding of cultural conventions and symbolic systems. Consequently, Jackson and Scott caution researchers to be wary of presupposing that meaning can be 'read off' from bodily responses - for example, an erect penis is conventionally read as signalling male desire but may not have the same meaning for the man experiencing it. It is precisely this uncertain and ambiguous relationship between bodily signs and the embodied experience of pleasure which is of interest here. The know-ability of what is experienced 'on the inside' is at the heart of concerns over the authenticity of female orgasm. Knowing and recognising the conventions through which orgasm is represented and experienced are essential cultural competencies for experiencing orgasm and recognising the orgasms of others. 
This paper, then, examines the interplay between the 'visual' and the 'embodied' in attempts to fix internal bodily states on the surface of the body in the context of uncertainty about the authenticity of women's orgasm. Since the visualising of orgasm is rarely an explicit focus of academic attention, I explore two areas in which the challenge to visually represent orgasm is particularly pertinent - the production and consumption of orgasm in pornography, and the performance of orgasm in everyday sexual interactions. These two realms are rarely brought into tension with one another, drawing as they do on different literatures and disciplinary concerns. However, they share in common a concern with producing orgasms which are see-able, knowable and recognisable by others (audiences and lovers), and with (visually) displaying or performing orgasm. Importantly, they also share a concern with authenticity - with establishing and maintaining the distinction between the 'real' and the 'fake'. Given the paucity of literature which specifically addresses the issue of visualising orgasm it is not possible to give an in-depth and detailed understanding of its representation in pornography or in everyday interaction. Instead, by placing these two realms in juxtaposition, my aim is to expose how they raise questions for each other about the processes of producing, interpreting, and recognising visual representations of orgasm as 'real'. I aim to problematise the distinction between the real and the fake by drawing attention to the discursive construction of authenticity as a meta-discourse which frames our understanding of sexual practices and their visual representation. Recently, Jagose (2010) argued for a 'queering' of our understanding of faking orgasm not as a problem - as a poor imitation of the real thing - but as a 'positive cultural practice', an 'invention' which offers 'a new disposition or way of managing oneself in sexual relations' (p. 535). Taking up Jagose's invitation, rather than considering the real and the fake as analytically or 
experientially distinct, I 'queer' authenticity by considering it as a necessarily contested meta-discourse which structures the interpretation and enactment of visual representations of orgasm.

I start by exploring pornographic representations of female sexual pleasure and orgasm. Although pornography is not the only place in which female orgasm is visualised (Jackson and Scott, 2007 give the example of Bernini's statue of Teresa of Avila, but we could equally point to contemporary advertisements), depicting sexual ecstasy is ostensibly the raison d'etre for pornography and the explicitness of the visual imagery is one of its defining features. Whilst I recognise that discussions of pornography in the singular tend to conflate genres, obscure queer or alternative pornographies, and blur different practices of production and consumption, my aim is not to present an analysis of specific pornographic representations. Rather I want to explore how 'showing' authenticity is a tension which is differently addressed in pornographies, and is an important element in (some) consumers engagement with these representations.

\section{Visualising orgasm in pornography}

Pornographies claim to offer images of 'real sex' as a key part of their appeal. Promising to freeze 'the action' as it happens, and capturing close-ups of (apparently) orgasmic bodies, are mechanisms for laying claim to authenticity (Paasonen 2006). Against this backdrop, the 'invisibility' of female orgasm presents a problem for pornography which necessitates a climax that is see-able. Drawing on Foucault's (1978) notion of scientia sexualis in which 
knowledge and power come together to classify the measurable 'truths' of sexual bodies and their pleasures, Williams (1989) argues that 1970s 'hard core' pornography emerges out of a desire to see and know the human body, and operates according to the 'principle of maximum visibility'. The moment of sexual ecstasy is captured by recording each involuntary spasm, and close attention to the detailed minutiae of bodily actions. The ejaculatory 'money shot' of the man represents the visible 'truth' of sexual pleasure, and simultaneously confirms the authenticity of the pornographic texts themselves (Paasonen 2006). The principle of maximum visibility necessitates a climax that is visible, a climax which reassures viewers that they are 'witnessing not the voluntary performance of feminine pleasure, but its involuntary confession' (Williams 1989, p. 50). With no equivalent to the ejaculatory money shot ${ }^{\mathrm{ii}}$, female porn stars are required to perform a 'frenzy of the visual' by representing a body out of control - a thrashing, writhing, moaning, screaming, performance of orgasmic ecstasy (Williams 1989). Yet, it is precisely this kind of excessive representation of female orgasm that has (over time) become clichéd, and raises doubts about the veracity of the sexual pleasure that it seeks to affirm.

\section{Amateurishly authentic}

Claims to offer a peep into 'real sex' are threatened by the increasing professionalization and commercialisation of porn's production - i.e. the use of celebrity performers, the silicone enhanced 'porn star look', polished but staged performances, and hackneyed plots and settings. The porn star's excessive orgasm has become marked as a parody or 
inauthentic simulation of the real thing. Against this back drop, porn scholars have (with varying degrees of criticality) examined new claims to 'realness' prompted by the rise in 'amateur' porn and the alternative forms of production and consumption enabled by new technologies. Russo (2007) argues that different genres of pornography typically claim to have a privileged relationship to the real along four different dimensions. Firstly, claims to 'realness of production' such as claims that un-simulated sexual acts have been recorded. For example, Messina argues that 'Realcore' is distinguished from other genres because it includes 'pictures of real people with real desires, having real sex in real places' (Messina n.d.). Secondly, claims to 'realness of representation' where images appear real due to their visual conventions. For example, 'amateur' content may be visually coded through grainy image quality, point-of-view shots, images of people with 'ordinary' or 'non-enhanced' bodies, the use of mundane settings, wide angle shots, long unedited segments, people talking to the camera, and a 'low-fi' style (Paasonen 2006, Russo, 2007, Messina, n.d.). Thirdly, claims to 'realness of reception' - that pornography produces real effects - arousal - in the viewer. Finally, claims to 'realness of social context' based on the relationship of production to real economic, political, and cultural processes. For example, the rise of peerto-peer exchanges and personal uploads has been heralded as a gift economy guided by the principles of pleasure which blurs the boundaries between production and consumption and removes amateur porn from the realms of commercial exploitation. Distinguishing between professionals (who are paid for their work) and amateurs (who do it for the love of it), is a mechanism for claiming authenticity. 
Amateur porn claims to be a 'better kind of porn' - one which is ethically produced and more real, raw, innovative and authentic (Paasonen 2010). For some claims that Netporn (pornographies specific to online platforms and networks) is distinct from porn-onthe-net (the re-presentation of familiar images and genres from print media and film on the internet, see Shah 2007) rest of the privileging of the amateur over the professional and marks a shift from a commercial to an exchange economy (Jacobs et al. 2007). Others point to the incorporation of the 'amateur' or 'reality' as a staple of mainstream porn-on-the-net as an attempt to reclaim realness from more alternative practices (Paasonen 2010, Ward 2010). For example, claims that amateur porn enables the representation of marginalized groups by showing bodies which are not stereotypically beautiful or sexy, overlook the fact that mainstream porn has always marketed such alternative bodies - albeit in the guise of a niche market (fat bodies, hairy bodies, old bodies, transsexual bodies, etc.). Similarly, claims that NetPorn offers alternative or subversive sexual performances are undermined by those who note that both the sexual practices and the ways they are displayed in amateur porn are subject to a normative 'pornoscript' which includes a prescriptive set of performances and camera shots which characterise pornographic visual production (van Doorn 2010). This pornoscript includes the fragmented presentation of both male and female body parts, showing genitals in extreme close up, the use of 'meat shots' (close up shots of penetration of vagina or anus with a penis typically recorded as if from the man's point of view), and the ejaculatory 'cum shot' as recurring themes. Paasonen (2010) points out that amateur producers 'are not merely expressing themselves, as a neoliberal discourse might have it, but commodifying themselves in relation to pornography as a genre and an industry' ( $p$. 1308). Amateur pornographers necessarily engage with (even as they try to subvert) the aesthetic conventions of mainstream porn. 
A detailed examination of the ways in which diverse genres of pornography lay claim to authenticity by positioning themselves differently in relation to these four dimensions of the real is beyond the scope of this paper. Like Paasonen, my concern here is not with establishing whether or not some kinds of pornography are more authentic, real, ethical or subversive than others, but with acknowledging that authenticity and artifice is one of the meta-discourses (cf. Boyle, 2011) through which pornographies market and construct themselves using a range of visual conventions. A growing body of work explores the ways in which the porn industry talks about itself and its product (Russo 2007, Paasonen 2010, Boyle 2011). Boyle (2011) demonstrates how women's abuse is marketed as part of (some) porn's appeal as their entry into the porn industry is presented as a remunerative solution to earlier abuse - a choice in which the 'skills' learned through abuse can be used to achieve money and celebrity. The victim-narrative is appropriated and creatively re-worked by the parts of the industry to curtail criticism. Similar flexibility is accorded to 'the real' which is prominent in a diverse range of 'alternative' pornographies (see, for example, Russo's interesting analysis of the 'real' in lesbian porn) as well as its mainstream counterpart. Claims to authenticity underpin the appeal of diverse pornographies and are implicated in the ways in which different genres attempt to mark out (and market) different territories of practice. Claims to represent real and authentic pleasure rest both on the visualising of bodies (i.e. the display of bodies, close-ups of genitalia), and on the visual practices through which these are displayed (i.e. shaky camera work, grainy images, poor sound quality, etc.). These representational conventions form part of how 'real' orgasms are made recognisable. 
Consuming pornographic 'reality'

The flexibility of authenticity as a meta-discourse in pornography raises questions about how consumers navigate this tricky discursive terrain. Although a concern with the real is often assumed to motivate consumers, the interpretative work of consuming pornography is severely under-researched. If, as Jackson and Scott suggest, interpreting fleshy bodies as orgasmic requires a set of cultural competencies, we would expect this to apply equally to the interpretation of bodies viewed on film or through a webcam. There is some, albeit limited, evidence which suggests that 'authenticity' structures the interpretative processes at work in consuming pornography. Young people report that pornography is a source of information about sexual anatomy and practices (Holland et al. 1998, Flood 2010) and a key place to learn about or to 'see' orgasms (Allen 2006), although they are often critical consumers of this material, believing it to be false, exaggerated or unrealistic (LöfgrenMårtenson and Månsson 2010). Some adult consumers use authenticity as a criterion for judging the quality of pornography, looking for 'genuine interest' where 'people are there because they want to be there', and evidence of real 'chemistry' between actors who are 'enjoying themselves' (McKee 2006). Scrutinising and assessing pornographic images for markers of authentic orgasms is a key part of the viewing experience:

[the actress] really obviously came and came a lot and I mean that wasn't just acting [...] she was obviously enjoying it and they both were and it was much more equal and that was good (Participant in McKee 2006, p. 527).

Conversely indicators of inauthentic pleasure such as 'women with really long fingernails fingering themselves' or 'porno kissing where they're just touching their tongues' were said 
to undermine the enjoyment of some female consumers (Parvez 2006, p. 617). These women closely scrutinised images to assess the authenticity of actresses' enjoyment, and this in turn was central to their own arousal and pleasure. Thus their enjoyment of the images (realness of reception) depends upon an interpretation of the performers as 'really' enjoying themselves not just appearing as if they are (realness of representation). Moreover, as reflexive consumers the women interpret the 'realness' of images in relation to their understanding of the context in which these images are produced; including a recognition of the exploitative processes of (some) porn production and the emotional and physical demands placed on performers - realness of social context (Parvez 2006). Whisnant (2010) argues that consumers may experience 'ethical qualms' about pornography 'and about themselves in so far as they enjoy such material' (p. 114). She describes the interpretative work which is cooperatively produced between the porn industry and male consumers as a kind of grooming which enables them to dismiss these qualms, and to read images which might otherwise be read as hostile or degrading as pleasurable. This includes drawing comparisons with other/worse consumers who view and enjoy other/worse material, claiming diminished responsibility since the consumer 'merely' watches rather than perpetrates the violence, and interpreting the action as something the women 'really' enjoys despite visual cues to the contrary. Of course, we should be wary of assuming that all consumers seek reassurance of women's authentic pleasure (some want reassurance of women's genuine pain or disgust, Whisnant 2010) or that all pornography depicts women in negative ways (or depicts women at all). Nonetheless, these studies expose how concerns with authenticity permeate individuals' readings of, and experiences with, pornography and how some viewers carefully scan images for evidence that feelings/action is faked. 
Consumers accrue cultural (and subcultural) competencies across a range of diverse genres and subgenres in how to read visual representations of embodied pleasure as real or fake.

\section{Visualising Orgasm in Everyday Sex}

A concern with knowing and visualising sexual pleasure is not limited to pornography. Pornography and sexology share a scientia sexualis fascination with making the 'truth' of sexual pleasure - specifically orgasm - visually demonstrable. When Masters and Johnson began observing and recording the physical responses associated with orgasm in the 1970s, they aimed to make visible the reality of sexual pleasure by inventing mechanisms for visually revealing bodily processes and responses. By identifying a checklist of visible signs which made orgasm unmistakable they assumed that these scientific truths would render the practice of faking orgasm redundant:

The obvious, rapid detumescence and corrugation of the areolae of the breasts and the definable contractions of the orgasmic platform in the outer third of the vagina remove any doubt as to whether the woman is pretending or experiencing orgasm (Masters and Johnson 1966, p. 134).

By identifying not only the internal physical changes that accompany orgasm (such as vaginal contractions), but also the external physical markers (such as changes in the breasts and a chest flush), Masters and Johnson offered a visible checklist of signs with which to verify the authenticity woman's orgasm. Yet, the ineffability of women's orgasm and men's inability to distinguish the real from the fake, remains a well-rehearsed dilemma in popular 
discourse - judging by the frequency with which this theme appears in both men's and women's lifestyle media. Master's and Johnson's checklist of visible signs is routinely repeated in popular culture and health advice as a means for men to establish whether their partner's orgasm is authentic:

Her external genitalia or vulva (including the clitoris, vaginal opening, and inner and outer lips or labia) become engorged (swollen) [...] She may become flushed, especially on her chest and neck, due to her blood vessels dilating (NHS Choices 2013).

Similarly, an article on the popular AskMen website subtitled 'How to Tell if She's Faking it' advises men to look for 'the tensing and releasing, in spasm, of many of the muscles throughout her body, including arms, legs, neck, and face. Even her toes will bend and arch forward' (Kerner 2013). Men are encouraged to read the 'inner' experience of orgasm from visible bodily changes, and to engage in a process of noticing and interpreting these bodily signs. 'Expert' knowledge offers the promise of being able to definitively distinguish between the real and the fake, and popular culture instructs men in how to develop the cultural competencies to spot the visual signs of a 'genuine' orgasm. Despite a dominant cultural story that improved scientific knowledge about sexual response and its visible signs will render orgasm visible and knowable, and despite the promise that scientific scrutiny will render faking orgasm obsolete (Jagose 2010), uncertainty and anxiety about the authenticity of female orgasm remains deeply embedded in the cultural imagination. 
In seeking to explore the gendered dynamics of faking orgasm in everyday heterosexual sex, feminists scholars have identified several key strands in the contemporary cultural construction of sexuality make the presence of orgasm a necessity, and provide fertile ground for faking. The orgasmic imperative (Potts 2002) creates a pressure to orgasm as the normal or expected outcome of sexual interaction (Fahs 2011) $)^{\text {iii; }}$ the ethic of reciprocity positions the mutual exchange of orgasms as right and fair (Braun et al. 2003); the coupling of sexual technique with masculinity such that a woman's orgasm is seen as affirmation of men's sexual skill (Fahs 2011, Roberts et al. 1995), and the expectation that women will sacrifice their own sexual needs in favour of caring for the emotions of their partners (Roberts et al. 1995). Together with the 'open secret' that heterosexual intercourse consistently fails to deliver the reciprocal orgasm - as evidenced by repeated social surveys showing that women are less likely to orgasm than men - the cultural conditions for faking to become acknowledged as widespread are established (Jagose 2010). Although this research addresses why women fake orgasm, it often fails to explore how women fake. Attention to the visible signs of orgasm produces the requirement to perform a 'noisy and exaggerated display' enacted through the body. In one of the few studies to ask how women fake orgasm (rather than why), Muehlenhard and Shippee (2010) found that most of their sample of 281 college students acted out an orgasm (78\% of men and $90 \%$ of women) in four different ways: a) bodily acting (using body movements such as moving or thrusting, freezing or clenching muscles); b) vocal acting (making sounds such as moaning or breathing faster or louder), c) verbal acting (saying words such as 'I'm coming'); and d) vague descriptions ('I just acted the same way [as when experiencing orgasm on a previous occasion]'). Faking orgasm is both embodied (done through the body) and a performance, which if done successfully will be indistinguishable from an authentic orgasm. Ironically, the 
same cultural imperatives which make orgasm faking orgasm a possibility (the orgasmic imperative, the ethic of reciprocity, the coupling of sexual technique with masculinity, and the uncertainty of women's sexual satisfaction) are the same imperatives which also make showing/displaying/performing a 'real' orgasm a necessity - and both are enacted through the same bodily performance. In order to produce a faked orgasm which can pass as authentic, women need considerable cultural competencies in the visualising of orgasmic experience.

\section{Performing the 'real'}

Up unto this point, I have demonstrated that authenticity is a meta-discourse which structures the reading of both pornography and sexual interactions, and that considerable interpretative work goes into reading the visual representation of orgasm in both contexts. Yet, for the most part the distinction between 'real' and 'fake' orgasms has been treated as relatively straightforward and is assumed to rest in the embodied experience of the individual. Whether an orgasm is real or fake rests of whether the individual 'feels' the orgasm or whether it is consciously performed. For example, 'pretending' orgasm is defined as: 'Acting like you were having an orgasm when you weren't actually having one, or saying that you had an orgasm when you really didn't' (Muehlenhard and Shippee 2010, p. 554). So, although a 'faked' orgasm may aspire to be visually indistinguishable from the real, the difference lies in the sensory experience of the woman. Here I want to complicate the distinction between real or fake orgasms by focusing on the visual production or 
representation of orgasm through the bodies of sex workers/porn performers and women in everyday sexual interactions. To shift from examining the reading/interpretation of visual representations, towards exploring the bodily processes of performing, displaying and experiencing orgasm.

To problematize the relationship between the real and the fake, the distinction between 'surface acting' and 'deep acting' as described in the theory of emotion work is of use. Emotion work was popularised by Hochschild $(1979,1983)$ to describe the labour that service workers engage in to manage their own and others' emotions ${ }^{\text {iv }}$. That is, the effort that people put in to trying to induce or inhibit feelings to make them 'appropriate' to the situation. Hochschild argued that such work was inherently ideological, since 'feeling rules' (rules governing what it is appropriate, expected or legitimate to feel in a given situation) outline the relationships, responsibilities and obligations that people have to each other in particular situations. Hochschild made a key distinction between 'surface acting' where people mask or disguise what they really feel (e.g. supressing disgust or feigning desire) in order to induce appropriate emotions in others, and 'deep acting' in which individuals work to actually feel differently themselves. Although originally developed to explain emotional management in commercial settings, emotion work has since been applied to the private sphere of intimate relationships. This work demonstrates how ideologies of love typically position women as primarily responsible for 'doing' emotional intimacy and men as unwilling or incapable of so doing, resulting in an unequal division of emotional labour (Duncombe and Marsden 1993, Erickson, 2005). 'Sex work' in which people work to 'bring their feelings more into line with how they suspect sex "ought to be" experienced' is one form of emotion work (Duncombe and Marsden 1996, p. 220). 
Faking orgasm is typically seen as a kind of 'surface acting' in which women disguise what they really feel (by suppressing disgust or boredom or by feigning desire and climax) in order to protect men from feeling sexually inadequate, or conversely to induce pleasure/arousal/orgasm in their partner (Roberts et al. 1995, Muehlenhard and Shippee 2010, Fahs 2011). Pretending to orgasm enables women to meet the 'feeling rules' governing heterosexual relationships which dictate that women should orgasm during sexual interactions, allowing them to avoid feelings of blame or inadequacy in relation to their own sexuality (Fahs 2011). Similarly, in commercial sex work, where the 'authenticity' of the workers pleasure is often a central part of what is being sold, women engage in surface acting to induce appropriate arousal and satisfaction in clients/audiences. Acting as if one experiences pleasure or desire, facilitates the illusion of authenticity while allowing women to protect themselves from the emotional and psychological demands of the work (Brewis and Linstead 2000, Sanders 2005, Bernstein 2007). The practice of faking, then, involves creating a credible visual representation of orgasm through the (surface of) the body.

In contrast, 'deep acting' requires women to actually feel pleasure and arousal rather than only pretending to do so. While the literature on deep acting is very sparse, there are hints that something more than surface acting might be at work. For example, couples in long term heterosexual marriages work to manage conflict about the frequency of sex arising from perceived sex differences in their 'natural' appetite for sex, by 'inducing desire' and making an effort to want sex more, or by repressing their desire for sex to match their partner (Elliott and Umberson 2008). Similarly, some women who identify as having sexual difficulties work to achieve 'normal' heterosexuality by changing their mental and physical 
responses to sexual practices (Cacchioni 2007). Often recommended by sex therapists and other experts, this is characterised by 'a quest to implement a certain skill and/or degree of concentration to manipulate the mind or the body' (Cacchioni 2007, p. 308). If this kind of work is undertaken to bring the body into line with feeling rules, can any pleasure which is experienced really be said to be genuine? Finally, some research findings related to faking orgasm muddy the distinction between the real and the fake. Some report experiences which were neither straightforwardly 'faking', nor 'real' or 'authentic' by responding positively to the statement 'I have never pretended to have an orgasm but I have done something similar', whilst others indicated that they had pretended to be more aroused or enthusiastic about sex than they really were suggesting some kind of continuum rather than a sharp distinction (Muehlenhard and Shippee 2010). Finally, some women report faking orgasm in order to increase their own arousal, excitement or interest in the sexual act (Cooper, Fenigstein and Fauber forthcoming).

Deep acting is also suggested in the context of commercial sex where some workers alter their feelings of disgust or attraction to clients. Sex workers who are aroused and attracted to a client, for example, report sometimes trying to turn what they perceive to be an unwanted or inappropriate emotion into something more acceptable (Sanders 2005). Moreover, some middle-class sex workers place a premium on ensuring that their labour feels meaningful to themselves, including 'trying to simulate - or even produce - genuine desire, pleasure and erotic interest for their clients' (Bernstein 2007, p. 484). Escoffier's (2007) work on gay male porn actors (although not drawing explicitly on emotion work), argues that pornography requires the performer to activate his own sexual fantasies in order to achieve erections and orgasms that can be visibly displayed. These real effects 
(erections and orgasms) make performing in pornographic films something more than only acting as though one was being sexual. Even if an actor's orgasm is 'real' (i.e. actually experienced) this does not mean that it is related to the action on the screen. This blurring of the boundaries between acting and experiencing problematises the idea that the body can be an unmediated source of knowing the 'truth' about orgasm.

\section{Concluding Remarks}

Gendered discourses serve to position the visual representation of women's sexual pleasure and orgasm as inherently problematic, uncertain and ambiguous in ways that are absent for men. By bringing literatures across a range of disciplinary boundaries to bear on the question of visualising orgasm, I have demonstrated that authenticity is a powerful discourse shaping the representation and experience of sexuality in diverse contexts. Challenging the idea that the distinction between the 'real' and the 'fake' is located within embodied experience and removed from social processes, this paper has begun to explore some of the interpretative and productive processes involved in producing and reading visual representations of orgasm in the context of a cultural investment in authenticity and the 'truth' of orgasm. Using the work of Jackson and Scott as a springboard I suggest that 'reading off' orgasms from the bodies of others - in pornography and everyday sexual interactions - requires considerable cultural competency. Moreover, by drawing on the theory of 'emotion work' I have argued that the distinction between acting and experiencing, between feeling orgasm or faking it, is far from clear cut since both require an 
embodied performance of orgasm which may be indistinguishable. Consequently, there is a need to interrogate how the real and the fake are constructed in specific contexts and, importantly, what ideological work this does. I suggest four areas for future research (although these are clearly not exhaustive).

Firstly, there is an urgent need for more critical exploration of the practices of porn consumption which go beyond simply cataloguing of how much or what type of porn people consume, and engages instead with how people actively interpret and make sense of pornographic texts and images (Attwood 2005, McKee 2006). The insightful work of Parvez (2006) and Whisnant (2010) begin to explore the interpretative work that consumers undertake, and the ways in which notions of realness and authenticity shape this experience. Parvez's work with female consumers indicates that they grapple with a desire to see images as authentic whilst being aware of the potentially exploitative and unpleasant realities of porn production. She points to consumers' awareness of the emotion work which actresses may engage in to appear as if they are enjoying themselves, but falls short of exploring the work that consumers might perform on themselves to suppress feelings of disgust in order to feel aroused. This idea is taken up by Whisnant who uses the notion of 'grooming' to describe how men may overcome uncomfortable feelings. I suggest that emotion work or 'deep acting' might sit alongside this grooming and usefully describe the interpretative work that consumers undertake to 'see' images as depicting 'real' pleasure for women and as therefore arousing. Moreover, Parvez's research showed that women with personal experience of poorly paid work and/or sexual abuse were particularly alert to exploitative working conditions within the porn industry, and this disrupted their ability to read the texts as pleasurable. Both Whisnant (2010) and Lindgren (2010) point to the 
homosociality of porn consumption - in which men watch porn collectively or interact online as members of a porn community - as shaping men's 'feeling rules' about what could or should be arousing. Porn consumers are reflexive beings and researchers need to treat them as such - this includes understanding how 'reading' pornography as 'real' is influenced by the presence of others, the intertextuality of different pornographic genres and the individual's own socio-biographical location.

Secondly, researchers should critically examine the ways in which discourses of the real and the fake structure public discourse about pornography and how pornography talks about itself. In her insightful analysis of the Cyber-Dyke network and its claims to offer 'real' lesbian porn, Russo (2007) pays careful attention to the visual and discursive codes through which 'realness' is represented. As she rightly points out, the fact that this discourse is drawn on by both commercial porn and alternative porn means that it is deserving of careful attention in order to identify the ideological work that is at play when images are described as unmediated. She argues that Cyber-Dyke is more real than commercial porn, not because of the innate sexuality of its producers (despite marketing itself as produced by lesbians), but because of the mobilization of recognizable markers of dyke subculture (e.g. butch bodies, tattoos and piercings, fetish attire) through which it participates in the dynamic production of identity, community and legitimacy (although this is not a 'reality' which all queer women will necessarily accept or identify with). In contrast, Whisnant (2010) points to the way in which heterosexual pornography shapes consumers perceptions by 'interpreting the images' - that is, by 'explaining in accompanying text (or DVD commentary) that a woman who appears not to be enjoying something actually loves it' ( $p$. 123). In other words, consumers are encouraged to overlook what they might in other 
circumstances interpret as women who are bored, sad or worried, as evidence of her eyes 'lighting up' with pleasure and enjoyment. That such diametrically opposed understandings of authenticity can be offered by pornographies which both draw on the discursive construction of the 'real' (and do so intertextually in relation to one another) necessitates further deconstruction.

Thirdly, the veracity of orgasm is also at stake in everyday interpersonal sexual relationships. Although we know something about the prevalence of faking, and about how people make sense of why they fake, we know little about how and when issues around faking become raised in sexual relationships. Research exploring the meaning-making processes through which lovers make sense of their own and each others' sexual responses, and when or how issues of veracity are negotiated would be a fruitful area of research. We have focused here on the veracity of female orgasm - since men's ejaculation is typically seen as unproblematically signifying orgasm and sexual satisfaction. Yet, research shows that a significant proportion of men also fake orgasm (Muehlenhard and Shippee 2010). Further research is needed to understand the interpersonal contexts in which the practice of faking becomes utilised, and how practices of visualising orgasm (making orgasm visible and interpreting these visual cues) are negotiated between couples. Importantly, we know little about when, how and in what contexts the veracity of orgasm is contested in interpersonal relationships, or how this is implicated in gendered power dynamics.

Finally, while this paper has identified that processes of showing and recognising orgasm through visual and embodied performances are at work in both pornographic representations and everyday life, it has only hinted at the relationship between these spheres. Attention to, and surveillance of, the body, specifically the appearance of the body 
- the way it moves, shakes, oozes, and undulates - is central to attempts to manage the uncertainties of sexual pleasure. While Jackson and Scott remind us that we cannot read off what someone is experiencing from their bodily display, this is of course the premise that producers and consumers of pornography, and lovers engaged in sexual interactions, work on. Pornography purports to offer a rare opportunity to see and hear 'real' orgasms as they are experienced 'real' people engaging in 'real' sexual activities. Yet, we know little about how these representations penetrate or are absorbed into the embodied experiences of sexual actors. Or, conversely, how embodied sexual interactions between lovers influence the interpretation of pornographic representations. Research could usefully explore how understandings of what 'fake' or 'real' orgasms look and sound like get negotiated among couples and under what circumstances.

In sum, if we take seriously Jackson and Scott's suggestion that all embodied experience is socially embedded, then the idea that the distinction between 'real' and 'fake' orgasms has any material foundation in sensory experience is disrupted, instead we are prompted to consider how and when this distinction has traction in the world and what the implications of this distinction are for gendered power relations, subjectivities and practices.

\section{References}

Allen, L., 2006. 'Looking at the real thing': Young men, pornography and sexuality education.Discourse: studies in the cultural politics of education, 27(1), 69-83. 
Attwood, F., 2005. What do people do with porn? Qualitative research into the consumption, use, and experience of pornography and other sexually explicit media. Sexuality and Culture, 9(2), 65-86.

Bernstein, E., 2007. Sex work for the middle classes. Sexualities, 10(4), 473-488.

Boyle, K. 2011. Producing abuse: Selling the harms of pornography. Women's Studies International Forum, 34, 593-602.

Braun, V., Gavey, N. and McPhillips, K., 2003.The 'fair deal'? Unpacking accounts of reciprocity in heterosex. Sexualities, 6(2), 237-261.

Brewis, J. and Linstead, S., 2000. Sex, work and sex work: Eroticizing organizations. London: Routledge.

Cacchioni, T., 2007. Heterosexuality and 'the labour of love': A contribution to recent debates on female sexual dysfunction. Sexualities, 10(3), 299-320.

Cooper, E.B., Fenigstein, A. and Fauber, R.L. forthcoming. The faking orgasm scale for women: Psychometric properties. Archives of Sexual Behavior. DOI: 10.1007/s10508-0130212-z.

Darling, C.A. and Davidson, J.K. 1986. Enhancing relationships: Understanding the feminine mystique of pretending orgasm. Journal of Sex and Marital Therapy, 12: 182-196. 
Duncombe, J. and Marsden, D., 1993. Love and intimacy: The gender division of emotion and 'emotion work': A neglected aspect of sociological discussion of heterosexual relationships. Sociology, 27(2), 221-241.

Duncombe, J. and Marsden, D., 1996. Whose Orgasm is this Anyway? Sex Work in LongTerm Couple Relationships.In J. Weeks and J.Holland, eds. Sexual Cultures. Basingstoke: MacMillan.

Elliott, S. and Umberson, D., 2008. The performance of desire: Gender and sexual negotiation in long term marriages. Journal of Marriage and the Family, 70, 391-406.

Erickson, R.J., 2005. Why emotion work matters: Sex, gender and the division of household labor. Journal of Marriage and the Family, 67(2), 337-351.

Escoffier, J., 2007. Scripting the sex: Fantasy, narrative and sexual scripts in pornographic films. In M. Kimmel, ed. The Sexual Self: The Construction of Sexual Scripts. Nashville, TN: Vanderbilt University Press, pp. 61-79.

Fahs, B, 2011. Performing sex: The making and unmaking of women's erotic lives. Albany: SUNY Press.

Flood, M. 2010. Young men using pornography. In K. Boyle, ed. Everyday Pornography. London: Routledge, 
Foucault, M., 1978. The history of sexuality. Vol. 1: An Introduction. Translated by Robert Hurley. New York: Pantheon Books.

Hite, S. 1976. The Hite Report. New York: Macmillan.

Hochschild, A.R., 1979. Emotion work, feeling rules and social structure. American Journal of Sociology, 85(3), 551-575.

Hochschild, A. R., 1983. The managed heart. Berkeley: University of California Press.

Holland, J., Ramazanoglu, C., Sharpe, S. and Thomson, R., (1998) The male in the head: Young people, heterosexuality and power. London: Tufnell.

Jackson, S. and Scott, S., 2007. Faking it like a woman? Towards an interpretive theorization of sexual pleasure.Body \& Society, 13(2), 95-116.

Jacobs, K., Janssen, M. and Pasquinelli, M., eds. 2007. C'Lick me: A netporn studies reader. Amsterdam: Institute of Network Cultures, 1-3.

Jagose, A. 2010. Counterfeit pleasures: fake orgasm and queer agency. Textual Practice, 24(3), 517-539. 
Kerner, I., 2013. Faking Orgasms: The Signs. Askmen.com. Available from:

http://uk.askmen.com/dating/love tip 500/584 faking-orgasms-the-signs.html [Accessed

21st March 2013].

Lindgren, S. 2010. Widening the Glory Hole: The Discourse of Online Porn Fandom.

Porn.com: Making Sense of Online Pornography. New York, NY: Peter Lang Publishing. 171 186.

Löfgren-Mårtenson, L. and Månsson, S.A., 2010. Lust, Love, and Life: A Qualitative Study of Swedish Adolescents' Perceptions and Experiences with Pornography. Journal of Sex Research, 47(6), 568-579.

Masters, W.H. and Johnson, V.E., 1966. Human Sexual Response. Boston: Little, Brown \& Co.

McKee, A., 2006. The aesthetics of pornography: the insights of consumers. Continuum: Journal of Media and Cultural Studies, 20(4), 523-539.

Messina, S. (n.d.) 'Realcore: The Digital Porno Revolution', URL (accessed 18 November 2013): http://realcore.radiogladio.it/

Muehlenhard, C.L. and Shippee, S.K., 2010. Men's and women's reports of pretending orgasm.Journal of Sex Research, 47(6), 552-567. 
NHS Choices. 2013. Sexual arousal in women. National Health Service, UK.

Available at: $\underline{\text { http://www.nhs.uk/Livewell/Goodsex/Pages/Sexualarousalinwomen.aspx. }}$

[Accessed 21st March 2013].

Paasonen, S., 2006. Email from Nancy Nutsucker: Representation and gendered address in online pornography. European Journal of Cultural Studies, 9(4), 403-420.

Paasonen, S., 2010. Labors of love: Netporn, Web 2.0 and the meanings of amateurism. New Media \& Society, 12(8), 1297-1312.

Parvez, Z.F., 2006. The labor of pleasure: How perceptions of emotional labor impact women's enjoyment of pornography. Gender \& Society, 20(5), 605-631.

Patton, C. 1989. Hegemony and orgasm - or the instability of heterosexual pornography. Screen, 20(1-2), 100-113.

Potts, A., 2002.The Science/Fiction of Sex: Feminist Deconstruction and the Vocabularies of Heterosex. London: Routledge.

Roberts, C., Kippax, S., Waldby, C. and Crawford, J., 1995. Faking it: The story of ‘Ohh!'.Women's Studies International Forum, 18(5/6), 523-532. 
Russo, J.L. 2007. 'The real thing': Reframing queer pornography for virtual spaces. In K. Jacobs, M. Janssen and M. Pasquinelli, eds. C'Lick Me: A Netporn Studies Reader. Amsterdam: Institute of Network Cultures, 239-251.

Sanders, T., 2005. 'It's just acting': Sex workers' strategies for capitalizing on sexuality. Gender, Work and Organization, 12(4), 319-42.

Schaefer, L.C. 1973. Women and sex: Sexual experiences and reactions of a group of thirty women as told to a female psychotherapist. New York: Pantheon.

Shah, N., 2007. PlayBlog: pornography, performance and cyberspace. In K. Jacobs, M. Janssen and M. Pasquinelli, eds. C'Lick Me: A Netporn Studies Reader. Amsterdam: Institute of Network Cultures, 31-44.

Van Doorn, N., 2010. Keeping it real: User-generated pornography, gender reification, and visual pleasure.Convergence, 16(4), 411-430.

Ward, A. E. 2010. Pantomimes of ecstasy: BeautifulAgony.com and the representation of pleasure. Camera Obscura 73, 25(1), 161-195.

Wiederman, M.W. 1997. Pretending orgasm during sexual intercourse: Correlates in a sample of young adult women. Journal of Sex and Marital Therapy, 23, 131-135. 
Williams, L., 1989. Hard core: Power, pleasure and the 'Frenzy of the Visible'. Berkeley:

University of California Press.

'I do not mean to suggest here that ejaculation is necessarily experienced as equivalent to orgasm for men, rather than ejaculation is culturally coded as the definitive signifier of male sexual pleasure regardless of whether it is experienced as such by individual men.

iifemale ejaculation does not seem to have the same authority as a signifier of female sexual pleasure and remains a marginal type of representation - for example, Paasonen (2006) found that only $6 \%$ of all 'money shots' in a sample of 366 unsolicited email (spam) messages advertising porn websites represented female ejaculation.

iii It could be argued that by focusing solely on orgasm that this paper perpetuates the orgasmic imperative and the elision of orgasm with sexual pleasure. However, I treat orgasm not solely as an embodied event, but as a set of social and sexual practices and discourses which is worthy of academic attention.

iv Hochschild used the term emotional labour to refer to this kind of work in a commercial setting, and emotion work to refer to similar processes in private, non-commercial settings. However, the terms are often used interchangeably. 